\title{
SpS1-Circumstellar disks \& their evolution: Dust
}

\author{
Carol A. Grady ${ }^{1}$ \\ ${ }^{1}$ Eureka Scientific \& Goddard Space Flight Center, Greenbelt, MD 20771 USA \\ email: Carol.A.Grady@nasa.gov
}

Circumstellar disks are an intrinsic part of star formation and are also where planets form, migrate, and where the materials capable of producing life-bearing worlds are produced. The most flamboyant signatures of the presence of disks are at infrared through millimeter wavelengths, where thermal emission from dust dominates the system light. The discovery and characterization of the emission from such disks has been a major activity for ground-based observatories and space missions (IRAS, ISO, MSX, AKARI, and Spitzer), and continues with the newest generation of infrared (IR) capabilities.

Spectral Energy Distributions (SEDs): The presence of excess light compared with the stellar photosphere not only demonstrates the presence of circumstellar material, but its shape offers insight into its spatial distribution of dust. There is a rapid decay in disk frequency over the first $5 \mathrm{Myr}$, consistent with the formation time for large asteroids, and dating of Saturn (Pascucci \& Tachibana 2009). Association average SEDs indicate grain growth to $10 \times$ interstellar sizes by 1-2 Myr (Bouwman et al. 2008; Sargent et al. 2009). The frequency of transitional disks, those with warm dust deficits relative to the average SEDS, indicating cleared lanes or cavities, increases over 2-5 Myr (Brown, 2009).

After 8-12 Myr, disk fractional IR luminosities $\left(\mathrm{F}_{I R E} / \mathrm{F}_{*}\right)$ are $\leqslant 10^{-3}$, and warm dust becomes rare (Moór et al. 2009). Overall $\mathrm{F}_{I R E} / \mathrm{F}_{*}$ drops with age, but with a large dispersion, permitting identification of debris disks which have undergone recent collisional activity (Wyatt 2008). Debris disk SEDs fall into two classes: those which can be fit by discrete blackbody components, indicating material confined to particle belts or rings, and those with more continuous dust distributions (e.g. disks) (Morales et al. 2009). Now the SED data can be combined with locations of Jovian-mass planets to map the planetary system architecture (Chen et al. 2008; Reidemeister et al. 2009).

Solid-State Features: Spectral features due to grains with radii $\leqslant 10 \mu \mathrm{m}$ are often seen. Absorption is typical of either embedded objects, or stars which are viewed with sight lines passing near the (high inclination) disk midplane. Otherwise, emission is seen.

Ices and Polycyclic Aromatic Hydrocarbons (PAHs): Water and simple molecular ices are seen in young stellar object (YSO) and high inclination disk spectra (Boogert et al. 2008; Pontoppidan et al. 2008; Gibb et al. 2004). Crystalline ice was detected in emission for a few stars by ISO (e.g. Malfait et al. 1999), and more recently in debris disks (Chen et al. 2008; Lisse et al. 2008). Water ice has been detected in reflectance spectra for a Herbig F star disk (Honda et al. 2009), but has not been seen in Herbig Ae star disks (Debes et al. 2008). Emission in a suite of mid-IR bands identified with PAHs is typical of Herbig Ae stars (Keller et al. 2008), is less common in T Tauri stars (Geers et al. 2006) and is not seen toward low-mass embedded YSOs (Geers et al. 2009). The PAH emission can extend over $\approx 100 \mathrm{AU}$ of the disk (Habart et al. 2006; Geers et al. 2007).

Dust: A wealth of silicate, oxide, and other dust features are commonly observed in the spectra of Herbig Ae, T Tauri, and some debris disks. Recent work in this area has focussed on establishing typical grain sizes, degree of silicate crystalinity, and chemistry for intermediatemass, T Tauri stars, and brown dwarfs using Spitzer IRS data. In addition to the distinct features, broader emission components can be identified using mineral libraries based on Solar System objects. There have been efforts to compare the mineralogy of the inner disks with outer disks from integrated light spectra (Sargent et al. 2009). Mid-IR interferometry demonstrates that silicate crystallinity drops with radius for Herbig Ae and bright T Tauri disks (Leinert et al. 2004; van Boekel et al. 2006). Chemical inventories of dust are now available for a few debris disks (Lisse et al. 2008).

Transient Phenomena: Variable solid-state emission has now been reported for Herbig Ae (Sitko et al. 2008) and T Tauri stars (Woodward et al. 2004). Increased silicate crystallinity 
during EX Lup's outburst demonstrates that significant grain processing occurs in the inner disks of pre-main-sequence stars (Ábrahám et al. 2009). Debris disk transient phenomena include large $\mathrm{F}_{I R E} / \mathrm{F}_{*}$, crystalline water ice, and species indicating hypervelocity impacts (Chen et al. 2008; Lisse et al. 2009).

The Future: Herschel, SOFIA, and SPICA will study crystalline water ice features, crystalline silicates, and hydrous silicates, providing an expanded set of mineral and ice diagnostics. ALMA observations will permit verification of disk structures inferred from modeling of SEDs. JWST, as well as providing high contrast imaging, will also enable spatially-resolved spectroscopic studies of small grains. In tandem with ground-based facilities, there will be new disk identifications from AKARI and WISE data, and more synoptic studies. The disk chemical inventories will facilitate systematic studies of whether stellar compositional peculiarities are inherited from their molecular cloud.

\section{References}

Ábrahám, P. et al., 2009, Nature 459, 224.

Boogert, A. et al., 2008, ApJ 678, 985.

Bouwman, J. et al., 2008, ApJ 683, 479.

Brown, J. et al., 2009, (this proceedings).

Chen, C. et al., 2009, arXiv 0906.3744.

Chen, C. et al., 2008, ApJ 689, 539.

Debes, J. et al., 2008, ApJ 673, L191.

Geers, V. et al., 2006, A\&A 459, 545.

Geers, V. et al., $2007 A \mathscr{E} A 476,279$.

Geers, V. et al., 2009, A\&A 495, 837.

Gibb, E. et al., 2004, ApJS 151, 35.

Habart, E. et al., 2006, A\&A 449, 1067.

Honda, M. et al., 2009, ApJ (Letters) 690, L110.

Keller, L. et al., 2008 ApJ 684, 411.

Leinert, Ch. et al., 2004, A\&A 423, 537.

Lisse, C. et al., 2008, ApJ 673, 1106.

Lisse, C. et al., 2009, arXiv 0906.2536.

Malfait, K. et al., 1999, A\&SA 345, L181.

Moór, A. et al., 2009, ApJ 700, L25.

Morales, F. et al., 2009, ApJ 699, 1067.

Pascucci, I. \& Tachibana, S. 2009, in Protoplanetary Dust: Astrochemical and Cosmochemical Perspectives Cambridge Planetary Science (No. 12), editors D. Apai and D. Lauretta, (Cambridge: Cambridge University Press) (in press).

Pontoppidan, K. et al., 2008, ApJ 678, 1005.

Reidemeister, M. et al., 2009, arXiv 0905.4688

Sargent, B. et al., 2009, ApJS 182, 477.

Sitko, M. et al., 2008, ApJ 678, 1070.

van Boekel, R. et al., 2006, Advances in Stellar Interferometry SPIE 6268, editors J. Monnier, M. Schöler, W. Danchi, E 13.

Woodward, C. et al., 2004 Debris Disks and the Formation of Planets, ASP Conf. Ser. 324 editors L. Caroff, L. J. Moon, D. Backman, E. Praton (ASP: San Francisco), p. 224.

Wyatt, M. C. 2008, ARAEA 46, 339. 